\title{
Myosin light chain kinase is responsible for high proliferative ability of breast cancer cells via anti- apoptosis involving $\mathbf{p} 38$ pathway
}

\author{
Wen-jing CUII, \#, Yi LIU1, \#, Xiao-lei ZHOU ${ }^{1}$, Feng-ze WANG ${ }^{1}$, Xiao-dong ZHANG ${ }^{2, *}$, Li-hong YE ${ }^{1, *}$ \\ ${ }^{1}$ Department of Biochemistry, the Key Laboratory of Bioactive Materials, Ministry of Education; ${ }^{2}$ Department of Cancer Research, Key \\ Laboratory of Molecular Microbiology and Technology of Ministry of Education, Institute for Molecular Biology and Biochemistry, Col- \\ lege of Life Sciences, Nankai University, Tianjin 300071, China
}

Aim: To investigate whether myosin light chain kinase (MLCK) contributed to the high proliferative ability of breast cancer cells. Methods: Soft agar colony formation on the MCF-7 and LM-MCF-7 cell lines was determined. The cell cycles of MCF-7 and LM-MCF-7 were detected using flow cytometry analysis. Western blot analysis was performed to detect the expression levels of p-ERK1/2, totalERK1/2, p-p38, total p38, p-JNK, total-JNK, survivin, Bcl-2, p-MLC, caspase-9, cleaved caspase-9, and MLCK. After treatment with adriamycin (ADR), ML-7 and SB203580, apoptosis was examined using flow cytometry analysis and Annexin V-FITC fluorescence microscopy.

Results: The breast cancer LM-MCF-7 cell line with high metastasis potential (a metastitic sub-clone of MCF-7) had higher anti-apoptosis ability relative to MCF-7 cells in response to adriamycin treatment (apoptosis rate: $6.76 \%$ vs $28.58 \%, P<0.05$ ). Moreover, the expression level of MLCK was upregulated and the level of phosphorylated p38 (p-p38) was decreased in LM-MCF-7 cells. Flow cytometry analysis showed that ML-7, selective inhibitor of MLCK, could induce apoptosis of the LM-MCF-7 cells, in which the level of $p-p 38$ was increased. Meanwhile, the expression levels of Bcl-2 and survivin were downregulated, while the caspase- 9 was upregulated suggesting that the cells were undergone apoptosis. Flow cytometry analysis showed that SB203580, an inhibitor of p38, abolished ML-7induced apoptosis, which resulted in the upregualtion of Bcl-2 and survivin, and downregulation of caspase-9, suggesting that Bcl-2, survivin and caspase-9 are downstream effectors of $\mathrm{p} 38$.

Conclusion: MLCK is responsible for high proliferative ability of breast cancer cells through anti-apoptosis, in which p38 pathway was involved.

Keywords: breast cancer; myosin light chain kinase; p38; cell proliferation; apoptosis; ML-7; Bcl-2 protein; survivin; caspases

Acta Pharmacologica Sinica (2010) 31: 725-732; doi: 10.1038/aps.2010.56; published online 10 May 2010

\section{Introduction}

Cytoskeleton proteins are key participants in the cellular progression to apoptosis, and the ability of the cytoskeleton to deform and reform is a crucial aspect of many cellular responses $^{[1]}$. Previous reports suggested that TNF-a triggers MLC (myosin light chain) phosphorylation-dependent endothelial actin cytoskeleton rearrangement with intercellular gaps and stress fiber formation, where the molecular motor underlying actin cytoskeletal changes is myosin ${ }^{[2,3]}$. Myosin II is the main nonmuscle class of myosin, consisting of two sets of myosin heavy chains $(200 \mathrm{kDa})$ and two sets of

\footnotetext{
\# These authors contributed equally to the article.

* To whom correspondence should be addressed.

E-mail yelihong@nankai.edu.cn (Prof Li-hong YE);

zhangxd@nankai.edu.cn (Prof Xiao-dong ZHANG)

Received 2010-01-31 Accepted 2010-04-08
}

MLC (16-20 kDa), and is regulated by MLC phosphorylation catalyzed by myosin light chain kinase (MLCK). Some reports have investigated the involvement of MLC phosphorylation in apoptotic membrane blabbing, a process distinct from the execution phase of apoptosis ${ }^{[4,5]}$. Moreover, MLCK plays a role in the regulation of epithelial cell survival ${ }^{[1]}$. Using pharmacological inhibition indicated that activation of the multifunctional MLCK by TNF-a is critical for the execution of the programmed cell death ${ }^{[6]}$. The organization and stiffness of the cytoskeleton are determined in large part by the forces generated by actin and myosin II $\left(\mathrm{MLC}_{20}\right)$, which is catalyzed by MLCK, stimulates the actin-activated, $\mathrm{Mg}^{2+}$-dependent ATPase activity of myosin II ${ }^{[7]}$. ML-7 [1-(5-iodonaphthalene1-sulfonyl)-1H-hexahydro-1,4-diazapine hydrochloride] is a cell-permeable, potent and selective inhibitor of MLCK $\left(K_{i}=0.3\right.$ $\mu \mathrm{mol} / \mathrm{L})$ that inhibits protein kinase $\mathrm{A}\left(K_{i}=21 \mu \mathrm{mol} / \mathrm{L}\right)$ and 
protein kinase $C\left(K_{i}=42 \mu \mathrm{mol} / \mathrm{L}\right)$ at higher concentrations ${ }^{[8]}$. Previous studies have showed that ML-7 induces apoptosis by itself and stimulates the ability of etoposide to induce apoptosis in mammary and prostate cancer cells. ML-7 also stimulates the ability of etoposide to prevent the growth of mammary and prostate tumours in mice and rats, respectively ${ }^{[6]}$. However, the mechanism by which ML-7 regulating apoptosis was seldom investigated.

Apoptosis, the most common form of programmed cell death in vertebrates, defines a type of regulated cell death associated with various morphological features that include cell shrinkage, nuclear/cytoplasmic fragmentation, and formation of dense bodies. Many studies have indicated that crucial apoptotic modulators are deregulated in metastatic cancer cells and support the hypothesis that suppression of apoptosis has a crucial role during the metastatic process ${ }^{[9,10]}$. Apoptosis can be induced via two distinct intracellular signaling pathways: the death receptor-mediated pathway and the mitochondrial activation-mediated pathway. The former pathway is triggered by ligation of death receptors such as Fas and TNFR1. In contrast to the death receptor pathway, the mitochondrial pathway responds to anticancer drugs and multiple classes of environmental stresses ${ }^{[11]}$. The released cytochrome $c$ participates in the process leading to caspase- 9 activation, followed by activation of caspase- 3 .

Cells are always required to integrate external stress signals and thus decide cell fates to die or survive on an ongoing basis. These fate decisions are made by a wide range of signaling pathways that are controlled by kinases. The mitogenactivated protein kinases (MAPKs) are the family of kinases that transduce signals from the cell membrane to the nucleus in response to a wide range of stimuli, including stress ${ }^{[12-14]}$. MAPKs are serine/threonine kinases that, upon stimulation, phosphorylate their specific substrates at serine and/or threonine residues. Conventional MAPKs consist of three family members: the extracellular signal-regulated kinase (ERK1/2); the c-Jun $\mathrm{NH}_{2}$-terminal kinase (JNK/SAPK1); and the p38 MAPK/SAPK2. The ERK pathway is activated by mitogenic stimuli, such as growth factors and cytokines. In contrast to ERKs, JNK and p38 MAPK are weakly activated by growth factors but respond strongly to a variety of stress signals including tumor necrosis factor, interleukin-1, ionizing and UV irradiation, and chemotherapeutic drugs ${ }^{[15]}$. Studies using the inhibitors of JNK and p38-MAPK have suggested that JNK and/or p38 MAPK activation is necessary for UV-, cytokine-, ceramide-, and chemotherapeutic drug-induced apoptosis ${ }^{[16,17]}$. The p38-MAPK signaling pathways are involved in a variety of cellular responses, and the outcomes of cellular response are varied and complicated. The p38 MAPK are phosphorylated and activated by dual kinases MKK3 and MKK6 at threonine and tyrosine regions. The p38-MAPKs control the function of transcription factors, kinases, or phosphatases such as ATF-2, MEF2, MAPKAPK, CDC25, or MSK1/ $2^{[15]}$.

However, the roles of MLCK involving high metastatic ability of breast cancer cells remain unclear. In the present study, we focused on the investigation of the roles of MLCK in anti- apoptosis using two parallel breast cancer cell lines, MCF-7 and LM-MCF-7, as models. Our finding shows that MLCK is responsible for high proliferative ability of breast cancer cells through anti-apoptosis involving p38 pathway. Potentially, MLCK may serve as a therapeutic target for breast cancer.

\section{Materials and methods \\ Cell lines and cell culture}

Breast cancer cell lines, such as MCF-7 and LM-MCF-7 ${ }^{[18]}$ (a metastatic sub-clone of MCF-7), were maintained in RPMI1640 medium supplemented with $10 \%$ fetal bovine serum and $100 \mathrm{U} / \mathrm{mL}$ penicillin, $100 \mathrm{U} / \mathrm{mL}$ streptomycin in humidified $5 \% \mathrm{CO}_{2}$ at $37^{\circ} \mathrm{C}$.

\section{Soft agar colony formation assay}

Anchorage-independent colony formation on these cell lines was determined as described previously ${ }^{[16]}$, with some modifications. Briefly, the cells were harvested and washed, and a total of $3 \times 10^{3}$ cells were resuspended in DMEM containing $0.3 \%$ agarose. The suspensions were cultured in 6-well plates above a layer of solidified 0.5\% agarose in DMEM with 10\% FCS. Soft agar colony formation assay was tested in triplicate for each cell line. After culturing for 3 weeks, representative fields of each cell line were photographed. Plates were stained with $0.5 \mathrm{~mL}$ of $0.005 \%$ Crystal Violet for $1 \mathrm{~h}$. The colonies were counted with a dissecting microscope.

\section{Flow cytometry analysis}

To detect the cell cycles of MCF-7 and LM-MCF-7, cells were harvested by trypsinization and washed twice with PBS. Washed cells were resuspended in 0.6 mL PBS ( $\mathrm{pH}$ 7.4), and fixed by addition of $1.4 \mathrm{~mL} 100 \%$ ethanol at $4{ }^{\circ} \mathrm{C}$ over night. Fixed cells were rinsed twice with PBS, and re-suspended in propidium iodine (PI) solution, including $50 \mu \mathrm{g} / \mathrm{mL}$ propidium iodide and $50 \mathrm{\mu g} / \mathrm{mL}$ RNaseA (Sigma, MO, USA) in PBS without calcium and magnesium, and then incubated at $37^{\circ} \mathrm{C}$ for $30 \mathrm{~min}$ in the dark. Stained cells were passed through nylon-mesh sieve to remove cell clumps and analyzed by a FACScan flow cytometer and Cell Quest analysis software (Becton Dickinson, San Jose, CA, USA). The cell proliferative index (PI) was calculated as the sum of the $S$ and $\mathrm{G}_{2} / \mathrm{M}$ phase cells, expressed as a fraction of the total cell population (PI $\left.=\left[\left(S+G_{2} / M\right) /\left(G_{0} / G_{1}+S+G_{2} / M\right)\right] \times 100 \%\right)^{[19]}$. For detecting the apoptosis of LM-MCF-7 after treating with $10 \mu \mathrm{mol} / \mathrm{L}$ adriamycin (ADR) or $20 \mu \mathrm{mol} / \mathrm{L}$ ML-7, two groups of LM-MCF-7 cells $\left(1 \times 10^{6}\right)$ were harvested by trypsinization and washed twice with PBS. The control group received no treatment. Then carrying out the experiment followed the aforementioned operation. Flow cytometry analysis was repeated three times independently.

\section{Western blot analysis}

Western blot analysis procedure was performed according to the reports ${ }^{[20,21]}$. After washing twice in cold PBS, the cells were lysed with ice-cold lysis buffer $(150 \mathrm{mmol} / \mathrm{L} \mathrm{NaCl}, 20$ $\mathrm{mmol} / \mathrm{L}$ Tris- $\mathrm{HCl}, \mathrm{pH} 7.4,0.1 \%$ SDS, $1.0 \%$ NP-40, 0.5\% Na- 
DOC, $0.2 \mathrm{mmol} / \mathrm{L}$ PMSF, and protease inhibitor cocktails). Lysates were centrifuged at $12000 \times g$ for $20 \mathrm{~min}$ and the supernatants were used as total cell lysates. Protein concentration was determined by Bradford protein assay (Bio-Rad, USA). A quantity of $30 \mu \mathrm{g}$ total protein per lane was separated by sodium dodecyl sulfate-polyacrylamide gel electrophoresis (SDS-PAGE) and transferred onto polyvinylidene fluoride (PVDF) membranes (Millipore). Membranes were blocked with $5.0 \%$ milk powder in $0.05 \%$ Tween-PBS, incubated with the antibodies, respectively, such as mouse anti-p-ERK1/2 (1:1000 dilution, Santa Cruz, USA), rabbit anti-total ERK1/2 (1:1000 dilution, Cell Signaling, USA), rabbit-anti-p-JNK (1:500 dilution, Santa Cruz), rabbit anti-total JNK (1:600 dilution, Santa Cruz), mouse anti-MLCK (1:500 dilution, Santa Cruz), rabbit anti-p-MLC (1:500 dilution, Santa Cruz), mouse anti-pp38 ${ }^{\text {Thr180/Tyr182 }}$ (1:500 dilution, NeoMarkers, USA), rabbit antitotal p38 (1:400 dilution, NeoMarkers, USA), mouse antisurvivin (1:1000 dilution, Chemicon, USA), mouse anti-Bcl-2 (1:500 dilution, NeoMarkers), rabbit anti-cleaved caspase-

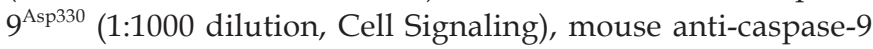
(1:500 dilution, Lab Vision, USA), mouse anti- $\beta$-actin (1:20 000 dilution, Sigma, MO, USA), at room temperature for $2 \mathrm{~h}$, and followed by a peroxidase-conjugated secondary antibody diluted in $0.3 \%$ BSA/Tween-PBS at room temperature for $1 \mathrm{~h}$. The bands were visualized using ECL reagent (Amersham biosciences, USA). All experiments were repeated three times. The Glyco Band-Scan software was used to show the contrast grey value to $\beta$-actin.

\section{Treatment with ADR, ML-7 and SB203580}

LM-MCF-7 cells were cultured in 6-well plates for $24 \mathrm{~h}$, and then the cells were re-cultured in serum-free medium for $12 \mathrm{~h}$ before treatment. LM-MCF-7 cells were treated with 10 $\mu \mathrm{mol} / \mathrm{L}$ adriamycin (ADR, Sigma) for flow cytometry analysis to examine apoptosis. In addition, $20 \mu \mathrm{mol} / \mathrm{L}$ ML-7 was added for 60 and $120 \mathrm{~min}$ for Annexin V-FITC fluorescence microscopy. Then, $20 \mu \mathrm{mol} / \mathrm{L}$ ML-7 was added for 30, 60, and 120 min for flow cytometry analysis to examine apoptosis, and 20 $\mu \mathrm{mol} / \mathrm{L}$ ML-7 was added for 15, 30, 60, and 120 min for Western blot analysis to detect p-MLC. Twenty $\mu \mathrm{mol} / \mathrm{L}$ SB203580 (Sigma) was added in LM-MCF-7 cells for 5, 20, and $40 \mathrm{~min}$, respectively, followed by Western blot analysis to detect the levels of p-p38, and the expression levels of survivin, Bcl-2, and caspase- 9 .

\section{Annexin V-FITC fluorescence microscopy}

LM-MCF-7 cells were divided into two groups which were individually treated with $20 \mu \mathrm{mol} / \mathrm{L} \mathrm{ML-7} \mathrm{for} 60$ and $120 \mathrm{~min}$. LM-MCF-7 cells receiving no ML-7 treatment were defined as control group. Cells were washed with phosphate buffered saline (PBS) and resuspended in lysis buffer $(10 \mathrm{mmol} / \mathrm{L}$ HEPES, pH 7.4, $140 \mathrm{mmol} / \mathrm{L} \mathrm{NaCl}$, and $2.5 \mathrm{mmol} / \mathrm{L} \mathrm{CaCl}_{2}$ ). Ten $\mu \mathrm{L}$ FITC-Annexin $\mathrm{V}$ and $5 \mu \mathrm{L}$ propidium iodide (PI) were added to a final concentration of $50 \mu \mathrm{g} / \mathrm{mL}$. The mixture was incubated for $15 \mathrm{~min}$ and then analyzed by fluorescent microscopy.

\section{Statistical analysis}

Statistical analysis was performed using Sigma Plot 10.0. Values were expressed as means $\pm S D$. Differences between groups were calculated with the Student's $t$ test. $P<0.05$ was defined as statistically significant.

\section{Results}

Expression levels of MLCK were inversely correlated with the levels of p-p38 in LM-MCF-7 cells with high proliferation ability

Previously, our laboratory established a metastatic sub-clone of breast cancer cell MCF-7 cell, termed LM-MCF-7 ${ }^{[18]}$. Here, we examined the growth capability of MCF-7 and LM-MCF-7 breast cancer cells. The soft agar clone formation assay showed that LM-MCF-7 had stronger clone formation ability than MCF-7 cells (Figure 1A, $P<0.05$ ). The flow cytometry analysis further showed that the proliferation index (PI) of LM-MCF-7 (PI: 36.21\%) is higher than that of MCF-7 cells (PI: 24.97\%) (Figure 1B, P<0.05 vs MCF-7 cells). Previously, we reported that MLCK was able to enhance growth and migration of breast cancer cells involving cross-talk with ERK1/2-MAPK in LM-MCF-7 cells ${ }^{[19]}$. In addition, we found that the levels of p-p38-MAPK were decreased in the cells ${ }^{[22]}$. In this study, Western blot analysis further confirmed the data (Figure 1C), suggesting that the expression levels of MLCK are inversely correlated with the levels of p-p38-MAPK. Thus, we suppose that p-p38 may be involved in the function of MLCK.

\section{LM-MCF-7 cells had higher anti-apoptotic capability than MCF-7 cell line}

Because ADR had the significant effect on inducing breast cancer cell apoptosis, we used $20 \mu \mathrm{mol} / \mathrm{L}$ ADR as an apoptosisinducer to treat the cells for $3 \mathrm{~h}$ to evaluate the anti-apoptotic ability between MCF-7 cells and LM-MCF-7 cells. Flow cytometry analysis revealed that LM-MCF-7 had lower apoptosis rate $(6.76 \%)$ than MCF-7 (28.58\%) (Figure $2 \mathrm{~A}, P<0.05$, LM-MCF-7 cells treated with $10 \mu \mathrm{mol} / \mathrm{L}$ ADR vs MCF-7 cells treated with $10 \mu \mathrm{mol} / \mathrm{L}$ ADR). Next, we examined the expression levels of several proteins related to cell apoptosis, such as survivin, Bcl-2 and caspase-9 between MCF-7 cells and LMMCF-7 cells by Western blot analysis. The results showed that the expression levels of survivin and Bcl-2 were upregulated in the cells, while the expression level of caspase- 9 was downregulated in the cells (Figure 2B), suggesting that LM-MCF-7 breast cancer cells own a higher anti-apoptosis capability than MCF-7 breast cancer cells.

\section{Disability of MLCK mediated by ML-7 resulted in apoptosis} involving activation of p38

Previous study has demonstrated that ML-7 induces apoptosis in prostate cancer cell ${ }^{[6]}$. Thus, in this study we used LM-MCF-7 cells as model to examine the effect of ML-7 on apoptosis. Annexin V-FITC fluorescence microscopy was applied to determine the incidence of apoptotic induced by $20 \mu \mathrm{mol} / \mathrm{L}$ ML-7 in LM-MCF-7 cells in a time course manner (Figure 3A). In addition, flow cytometry analysis showed that the treatment with $20 \mu \mathrm{mol} / \mathrm{L}$ ML-7 was able to induce apop- 

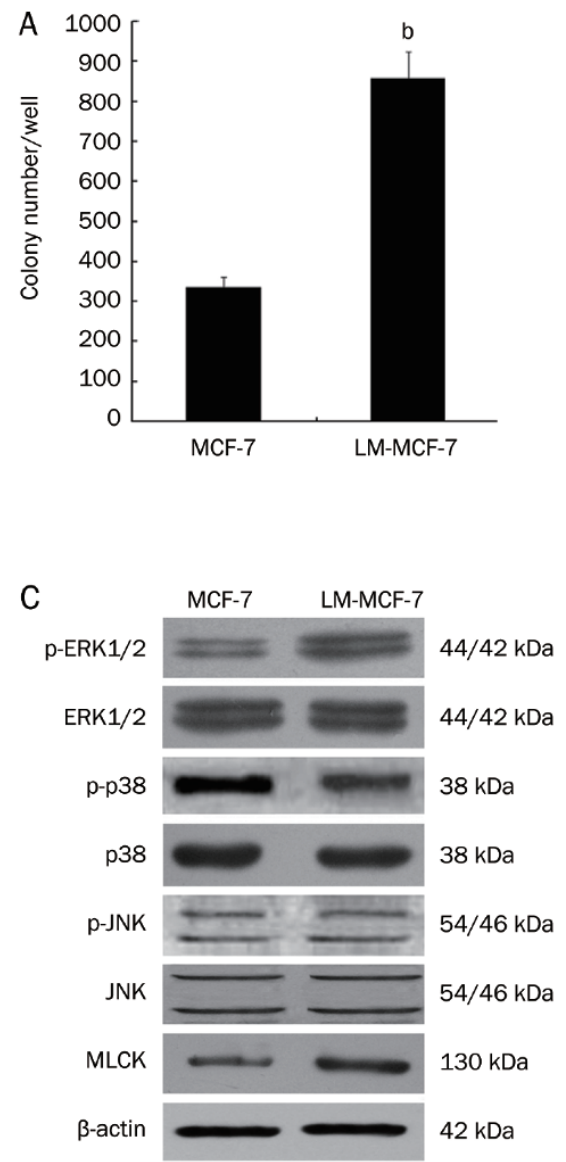

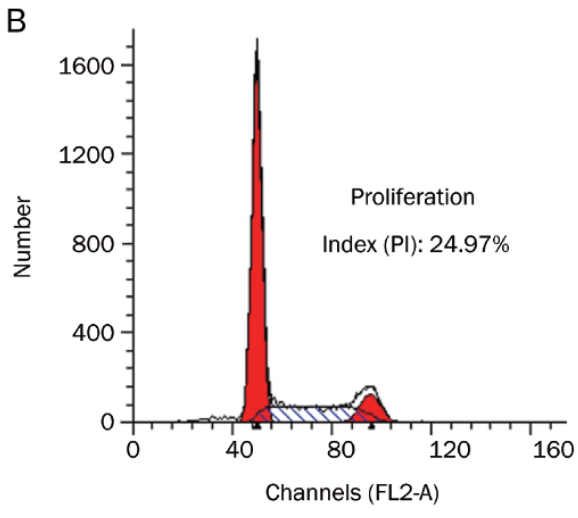

MCF-7

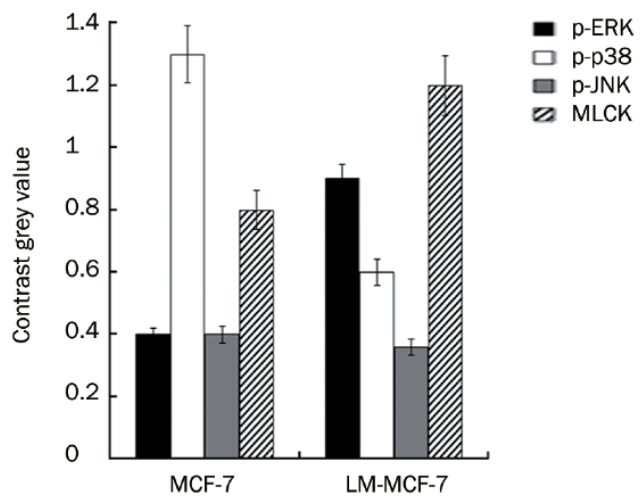

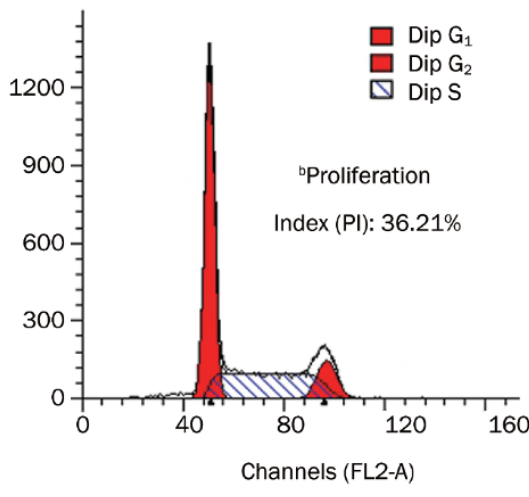

LM-MCF-7

Figure 1. Expression level of MLCK was inversely correlated with the levels of p-p38 in LM-MCF-7 cells with high proliferation ability. (A) The histogram showed the colony number of MCF-7 and LM-MCF-7 cells per well in soft agar for reflecting the growth capability between MCF-7 and LM-MCF-7 ( $\left.{ }^{\mathrm{b}} P<0.05\right)$. Bars indicate the standard deviations. (B) Proliferation capability between MCF-7 and LM-MCF-7 was tested by flow cytometry analysis. The proliferation index (PI) was calculated by the formula in Material and method section $\left({ }^{\mathrm{b}} \mathrm{P}<0.05\right.$ vs MCF-7 cells). (C) The expression levels of $\mathrm{p}$-ERK1/2, total-ERK1/2, p-p38, total p38, p-JNK, total-JNK and MLCK between MCF-7 and LM-MCF-7 cells were examined by Western blot analysis. Histogram shows the contrast grey value to $\beta$-actin by applying Glyco Band-Scan software.

tosis in the LM-MCF-7 cells in a time course manner (Figure $3 \mathrm{~B}, P<0.05$, vs Mock). Western blot assay demonstrated that the level of phosphorylated MLC (p-MLC) were decreased by treatment with $20 \mu \mathrm{mol} / \mathrm{L}$ ML-7 in a time course manner (Figure 3C), suggesting that the treatment resulted in the disability of MLCK in function. Meanwhile, the level of p-p38 was increased in the event, which supported that p-p38 was involved in the induction of apoptosis mediated by ML-7. We observed that the expression levels of survivin, Bcl-2 and full-length caspase- 9 were downregulated and the expression level of cleaved caspase- 9 was upregulated, which further supported that the cells were undergone apoptosis. Thus, our finding indicates that disability of MLCK mediated by ML-7 results in apoptosis of LM-MCF-7 cells involving the activation of p38-MAPK.

\section{Activation of p38-MAPK was responsible for the apoptosis} mediated by ML-7

To further confirm the role of p-p38 in apoptosis mediated by ML-7, we used the inhibitor of p38 ( $20 \mu \mathrm{mol} / \mathrm{L} \mathrm{SB203580)}$ to block the induction of apoptosis mediated by ML-7 in LMMCF-7 cells. Flow cytometry analysis showed that the treatment with $20 \mu \mathrm{mol} / \mathrm{L}$ SB203580 could abolish the apoptosis induced by $20 \mu \mathrm{mol} / \mathrm{L}$ ML-7 (Figure $4 \mathrm{~A}, P<0.05$ vs only 20 $\mu \mathrm{mol} / \mathrm{L}$ ML-7 treatment). The treatment with $20 \mu \mathrm{mol} / \mathrm{L}$ ML-7 could decrease the level of p-MLC and expressional levels of survivin and Bcl-2 in a time course manner $(5 \mathrm{~min}, 20 \mathrm{~min}$, and $40 \mathrm{~min})$. Conversely, the treatment could increase the level of p-p38 and expressional levels of cleaved caspase-9 (Figure 4B). Furthermore, Western blot analysis provided evidence that the downregulated survivin and Bcl-2 proteins, and the increased p-p38 and cleaved caspase- 9 protein, mediated by the treatment with $20 \mu \mathrm{mol} / \mathrm{L}$ ML-7, could be rescued by the treatment with $20 \mu \mathrm{mol} / \mathrm{L}$ SB203580 in a time course manner (Figure 4B), suggesting that survivin, Bcl-2 and caspase- 9 are downstream effecters of p38. Thus, we conclude that the activation of p38MAPK is responsible for the apoptosis mediated by ML-7.

\section{Discussion}

Breast cancer is the most common cancer in the world, and 

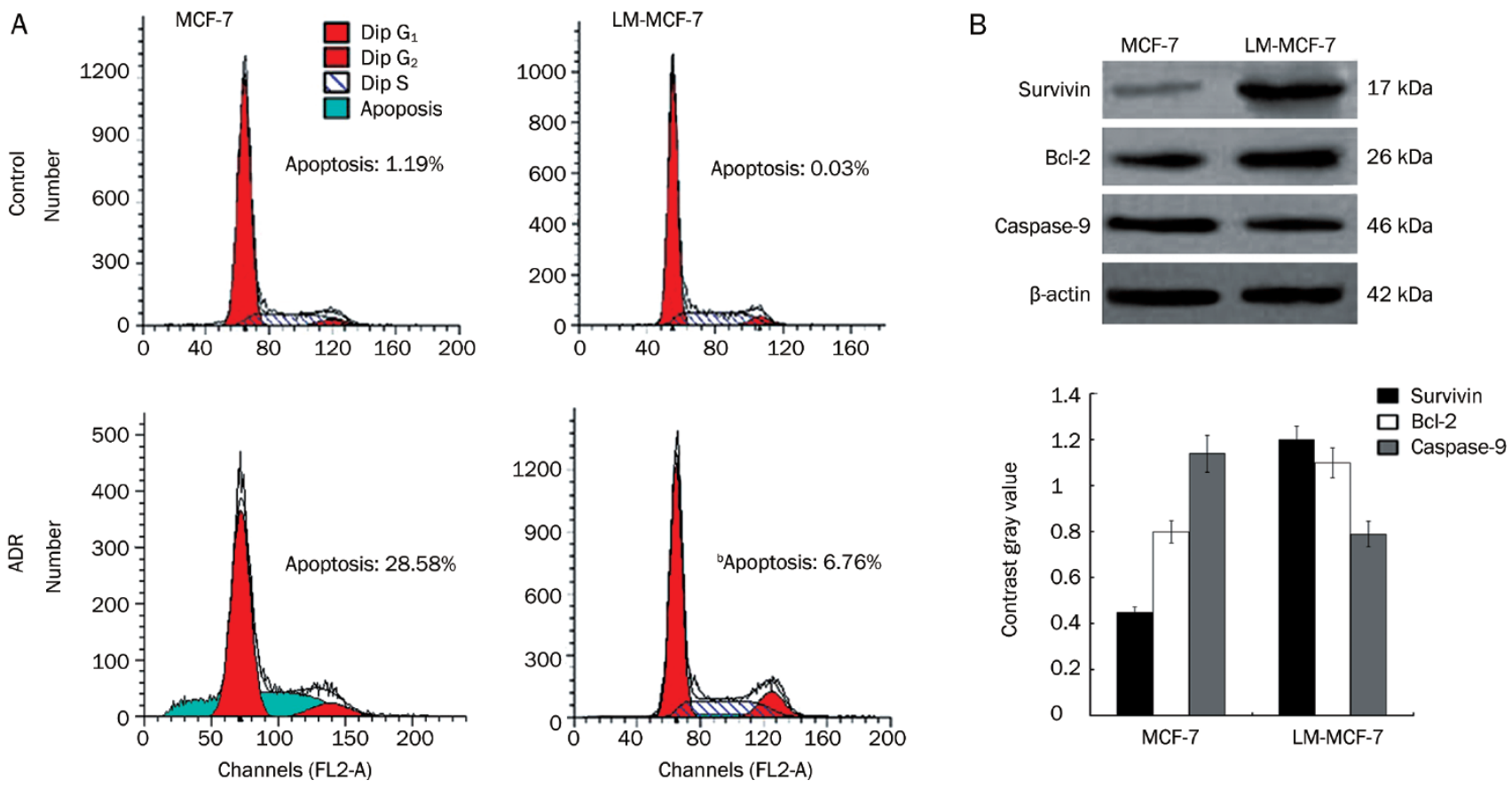

Figure 2. LM-MCF-7 cells had higher anti-apoptotic capability than MCF-7 cell line. (A) Effect on apoptosis that induced by ADR between MCF-7 and LM-MCF-7 cells was examined by flow cytometry analysis $\left({ }^{b} P<0.05\right.$, LM-MCF-7 cells treated with $10 \mu \mathrm{mol} / \mathrm{L}$ ADR vs MCF-7 cells treated with $10 \mu \mathrm{mol} / \mathrm{L}$ ADR, Student's $t$ test), in which the PI staining and analysis of sub- $\mathrm{G}_{1}$ proportion were used. (B) The expression levels of anti-apoptosis associated proteins survivin, Bcl-2, and apoptosis associated protein caspase-9 in MCF-7 and LM-MCF-7 cells were examined by Western blot analysis. Histogram shows the contrast grey value to $\beta$-actin by applying Glyco Band-Scan software.

remains the second leading cause of cancer related deaths in females. The development and continued growth of breast cancers involves altered rates of cell proliferation. In early breast cancer, measurement of proliferation can be used in conjunction with tumor size, grade, nodal status and steroid receptor status as a prognostic indicator ${ }^{[23]}$. Dys-regulation of apoptosis occurs commonly in a wide variety of human malignances. The failure of cancer cells to undergo apoptosis induced by anti-neoplastic agents is a major problem in cancer therapy. The aggressiveness of tumors is, in part, because of their acquired resistance to apoptosis ${ }^{[24-26]}$. Previously, we established a breast cancer cell line LM-MCF-7, which was derived from lung metastasis of severe combined immunodeficiency (SCID) mouse transplanted with MCF-7 cells. The LM-MCF-7 cells were injected subcutaneously into SCID mice, metastasized to lung, kidney, spleen, bone marrow, lymph node and heart extensively ${ }^{[27]}$. The LM-MCF-7 breast cancer cell line has higher proliferation and metastasis ability than its parallel cell MCF-7. We previously reported that cross-talk of ERK1/2-MAPK with MLCK contributed to the proliferation and migration in LM-MCF-7 breast cancer cells. Dramatically, the phosphorylated p38-MAPK in LM-MCF-7 was decreased relative to MCF-7 cells ${ }^{[22]}$. Thus, we supposed that p-p38 might be involved in the function of MLCK in high malignant phenotype breast cancer cell line LM-MCF-7. Herein, we engaged in the investigation focusing on the problem.

We firstly compared the growth and proliferation capability between MCF-7 and LM-MCF-7 cells to confirm the high malignant phenotype of LM-MCF-7 by soft agar colony formation assay and flow cytometry analysis. The results suggested that the growth and proliferation capability of LM-MCF-7 cells was stronger than MCF-7 cells (Figure 1A-1B). According to our previous study of expression of MAPKs in LM-MCF-7 $7^{[22]}$, we compared the MAPKs and MLCK expression between MCF-7 and LM-MCF-7 cells. We found that levels of p-p38 were decreased in LM-MCF-7 cells, which were inversely correlated with the expression levels of MLCK. Previous study demonstrated that the inhibition of MLCK with specific inhibitor ML-7 was able to induce cancer cell apoptosis ${ }^{[7]}$. Thus, we supposed that overexpression of MLCK could be associated with the levels of p-p38 in anti-apoptosis in LM-MCF-7 breast cancer cells.

It was known that the ADR had the significant effect on inducing breast cancer cell apoptosis ${ }^{[28]}$. Therefore, to confirm the hypothesis above, we evaluated the anti-apoptotic ability using ADR between MCF-7 and LM-MCF-7 cells. We found that LM-MCF-7 cells showed much more anti-apoptotic ability than MCF-7 cells after induction of ADR. Next, we manifested that the expression level of anti-apoptosis associated protein survivin, Bcl-2 were higher than that in MCF-7 cells, and the expression level of apoptosis associated protein caspase-9 was lower in LM-MCF-7 cells (Figure 2A-2B). Thus, all of the evidence pointed to a fact that the anti-apoptotic ability of LM-MCF-7 cells was higher than MCF-7 cells. It had been reported that inhibition of MLCK induced apoptosis in cancer cells ${ }^{[7]}$. In this study, we demonstrated that the inhibition of 

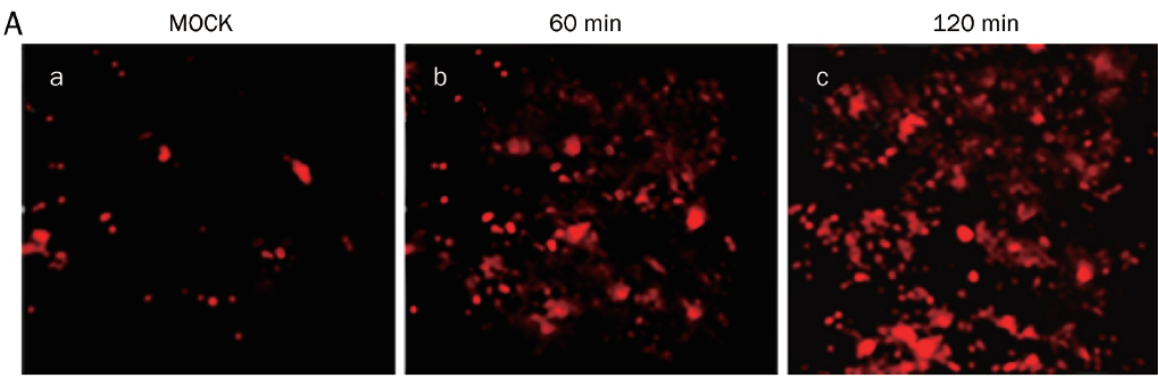

LM-MCF-7 (treated with $20 \mu \mathrm{mol} / \mathrm{L} \mathrm{ML-7)}$

B

Treated with $20 \mu \mathrm{mol} / \mathrm{L} \mathrm{ML}-7$
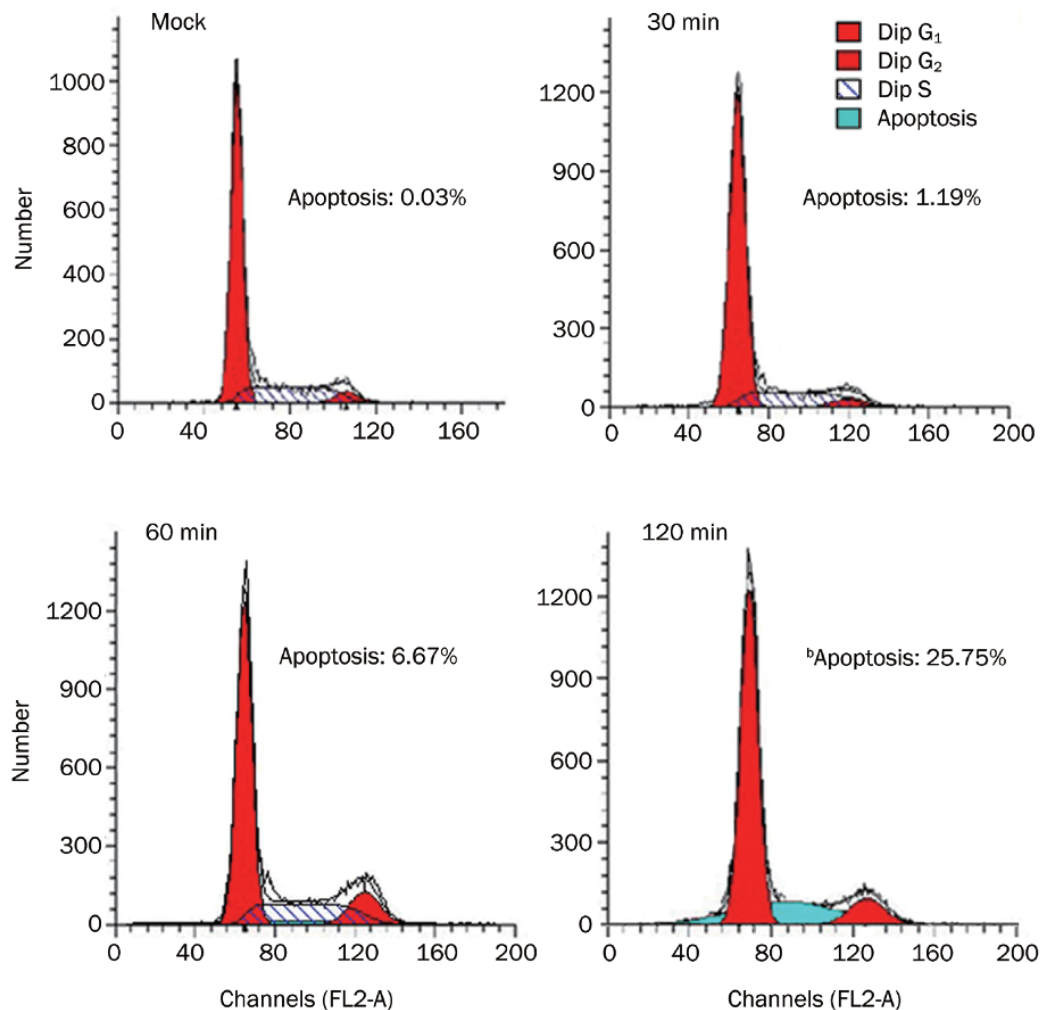

C ML-7 $(20 \mu \mathrm{mol} / \mathrm{L}) \quad 0 \quad 15 \quad 30 \quad 60 \quad 120(\mathrm{~min})$
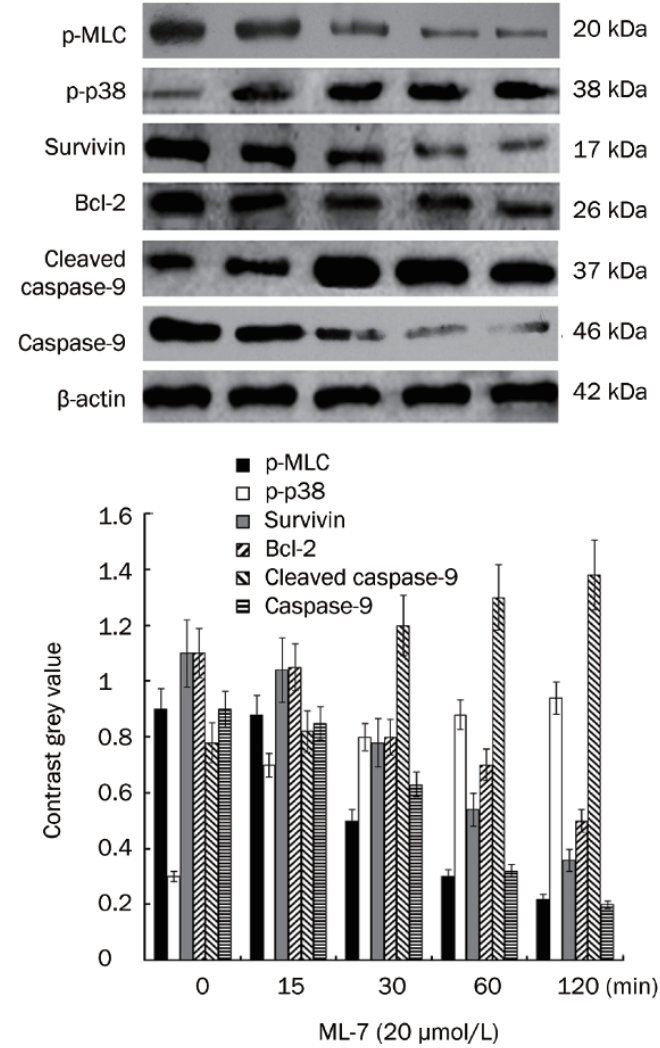

Figure 3. Disability of MLCK mediated by ML-7 resulted in apoptosis involving activation of p38. (A) ML-7-induced apoptosis of LM-MCF-7 cells was measured by Annexin V-FITC analysis using fluorescent microscopy. LM-MCF-7 cells untreated with ML-7 served as control. (B) ML-7-induced apoptosis of breast cancer LM-MCF-7 cells was examined by flow cytometry analysis. LM-MCF-7 cells were treated with $20 \mu \mathrm{mol} / \mathrm{L}$ ML-7 for 30,60 , and 120 min, respectively. Mock, LM-MCF-7 cells was not treated with $20 \mu \mathrm{mol} / \mathrm{L} \mathrm{ML-7}$, served as control $\left({ }^{\mathrm{b}} \mathrm{P}<0.05\right.$, vs mock). The PI staining and analysis of proportion of sub- $\mathrm{G}_{1}$ were used. (C) The levels of p-p38 MAPK and the expression levels of survivin, Bcl-2, cleaved caspase-9 and caspase-9 were examined by Western blot analysis in LM-MCF-7 cells after treatment with $20 \mu \mathrm{mol} / \mathrm{L} \mathrm{ML-7} \mathrm{for} \mathrm{15,} \mathrm{30,} \mathrm{60,} \mathrm{and} 120$ min, respectively. Histogram shows the contrast grey value to $\beta$-actin by applying Glyco Band-Scan software.

MLCK mediated by ML-7 resulted in apoptosis of LM-MCF-7 cells (Figure 3A-3B), which was consistent with the previous study in prostate cells ${ }^{[6]}$. In order to detect the mechanism of apoptosis induced by ML-7, we detected the expression levels of p-p38, survivin, Bcl-2 and caspase-9 when LM-MCF-7 cells were treated with ML-7. Western blot analysis showed that $20 \mu \mathrm{mol} / \mathrm{L}$ ML-7 stimulated the activation of p38-MAPK and upregulated the expression of cleaved caspase-9, but it downregulated the expression of survivin, full-length caspase- 9 and
Bcl-2 (Figure 3C). These findings suggest that the inhibition of MLCK by ML-7 is likely able to induce apoptosis through p38MAPK in LM-MCF-7 breast cancer cells.

The stress-activated protein kinases p38-MAPK is believed to play important roles in apoptosis ${ }^{[29]}$. Studies using the inhibitor of p38-MAPK have suggested that p38-MAPK activation is necessary for UV-, cytokine-, ceramide-, and chemotherapeutic drug-induced apoptosis ${ }^{[30]}$. Therefore, p38 has recently gained attention as a tumor suppressor. A comparison of 20 

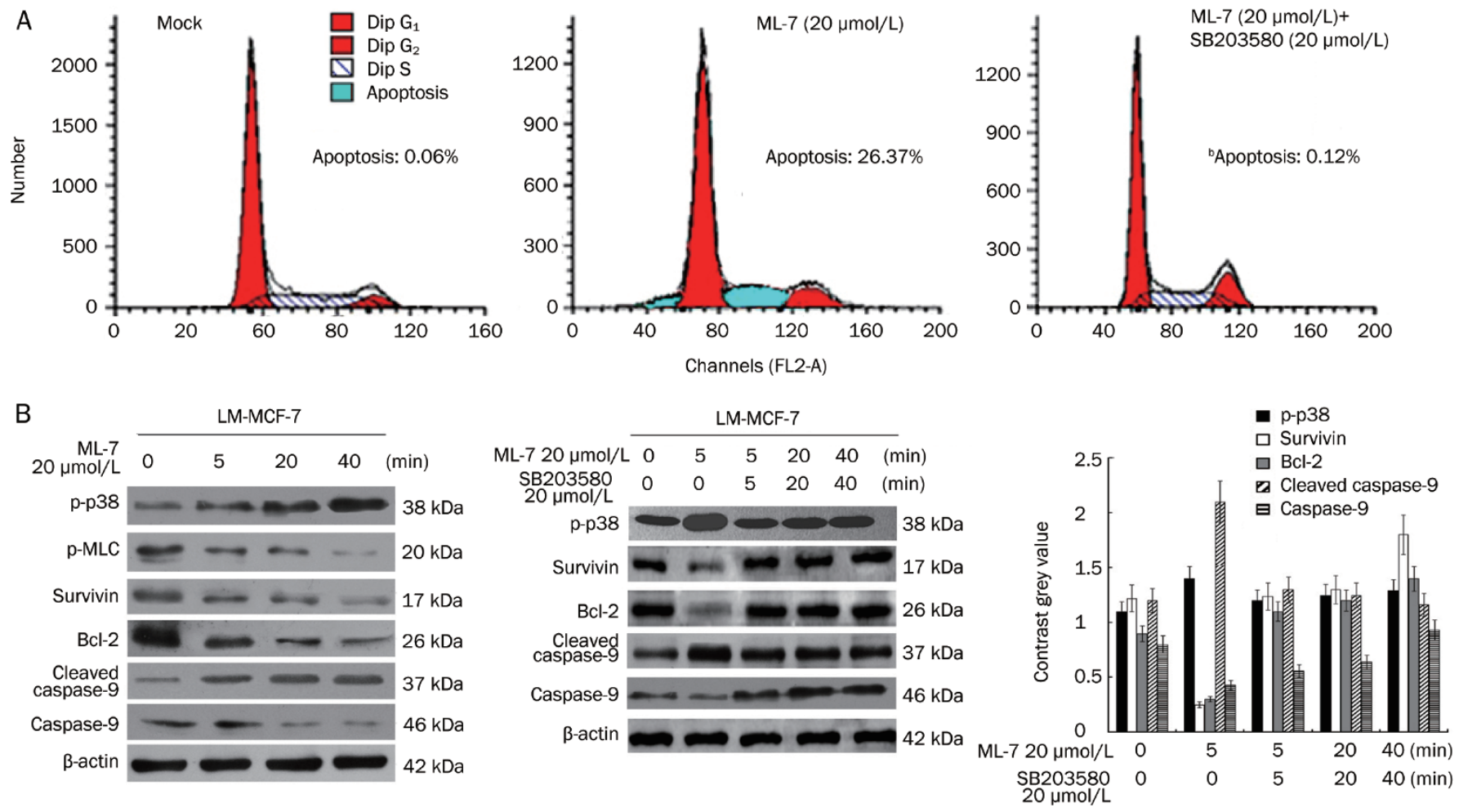

Figure 4. Activation of p38-MAPK was responsible for the apoptosis mediated by ML-7. (A) Flow cytometry analysis showed the effect of SB203580 on ML-7-induced apoptosis in LM-MCF-7 cells. Treatment with $20 \mu \mathrm{mol} / \mathrm{L}$ SB203580 was able to abolish the apoptosis induced by $20 \mu \mathrm{mol} / \mathrm{L}$ ML-7 in LMMCF-7 cells ( ${ }^{\mathrm{b} P}<0.05$, vs only $20 \mu \mathrm{mol} / \mathrm{L}$ ML-7 treatment). The PI staining and analysis of proportion of sub-G $\mathrm{B}_{1}$ were used. (B) The expression levels of p-MLC, p-p38, survivin, Bcl-2, cleaved caspase-9, and caspase-9 were examined by Western blot analysis in LM-MCF-7 cells after treatment with 20 $\mu \mathrm{mol} / \mathrm{L} \mathrm{ML-7}$ alone or with both $20 \mu \mathrm{mol} / \mathrm{L} \mathrm{ML-7}$ and $20 \mu \mathrm{mol} / \mathrm{L} \mathrm{SB} 203580$ for 5, 20, and $40 \mathrm{~min}$, respectively.

human hepatocellular carcinomas with adjacent nonneoplastic tissue showed that p38 was significantly less active in tumors. Additionally, the larger the tumor, the less p38 activity was detected $^{[31]}$. These studies indicate that the sustained p38 activity is consistent with dormancy behavior and inconsistent with proliferation and growth of primary tumors in vivo. In our study, we demonstrated that ML-7-induced apoptosis could be rescued by $20 \mu \mathrm{mol} / \mathrm{L}$ SB203580 (a p38-MAPK inhibitor). As control, the treatment with $20 \mu \mathrm{mol} / \mathrm{L} \mathrm{ML-7}$ alone was also able to inhibit the expression of survivin, $\mathrm{Bcl}-2$, and to activate p38 and caspase-9. Meanwhile, the stimulation of p-p38 and increased cleaved caspase- 9 mediated by $20 \mu \mathrm{mol} / \mathrm{L}$ ML-7 could be effectively inhibited by $20 \mu \mathrm{mol} / \mathrm{L}$ SB203580, whereas the inhibition of survivin and Bcl-2 mediated by ML-7 could be rescued by $20 \mu \mathrm{mol} / \mathrm{L}$ SB203580 (Figure 4A-4B), suggesting that survivin, Bcl-2 and caspase- 9 are downstream effectors of p38-MAPK. These results demonstrate that the apoptosis induced by ML-7 is involved in p38-MAPK signal pathways in breast cancer cells.

Taken together, we demonstrate that high malignant potential breast cancer LM-MCF-7 cells display powerful antiapoptotic ability than the breast cancer MCF-7 cells because of higher expression levels of MLCK. Inhibition of MLCK mediated by ML-7 is able to induce apoptosis in LM-MCF-7 cells, and alters the p38-MAPK level. Thus, we conclude that MLCK is responsible for high proliferative ability of breast cancer cells through anti-apoptosis involving p38 pathway. Potentially, MLCK may serve as a therapeutic target for breast cancer.

\section{Acknowledgements}

This work was supported from National Basic Research Program of China (973 Program, No 2007CB914804, № 2007CB914802, № 2009CB521702) and National Natural Scientific Foundation of China (№ 30770826).

\section{Author contribution}

Prof Li-hong YE and Prof Xiao-dong ZHANG designed the research, analyzed the data, wrote the manuscript and revised the paper; Wen-jing CUI and Yi LIU performed the research; Xiao-lei ZHOU and Feng-ze WANG help to perform part of the research.

\section{Abbreviations}

MLCK, myosin light chain kinase; p-MLC, phosphorylated myosin light chain; MAPK, mitogen-activated protein kinase; ERK, extracellular signal-regulated kinase; p-p38, phosphorylated p38; ADR, adriamycin; SCID, severe combined immunodeficiency. 


\section{References}

1 Connell LE, Helfman DM. Myosin light chain kinase plays a role in the regulation of epithelial cell survival. J Cell Sci 2006; 119: 2269-81.

2 Petrache I, Crow MT, Neuss M, Garcia JG. Central involvement of Rho family GTPases in TNF-alpha-mediated bovine pulmonary endothelial cell apoptosis. Biochem Biophys Res Commun 2003; 306: 244-9.

3 Petrache I, Verin AD, Crow MT, Birukova A, Liu F, Garcia JG. Differential effect of MLC kinase in TNF-alpha-induced endothelial cell apoptosis and barrier dysfunction. Am J Physiol Lung Cell Mol Physiol 2001; 280: L1168-78.

4 Petrache I, Birukov K, Zaiman AL, Crow MT, Deng H, Wadgaonkar R, et al. Caspase-dependent cleavage of myosin light chain kinase (MLCK) is involved in TNF-alpha-mediated bovine pulmonary endothelial cell apoptosis. FASEB J 2003; 17: 407-16.

5 Wadgaonkar R, Linz-McGillem L, Zaiman AL, Garcia JG. Endothelial cell myosin light chain kinase (MLCK) regulates TNFalpha-induced NFkappaB activity. J Cell Biochem 2005; 94: 351-64.

6 Gu LZ, Hu WY, Antic N, Mehta R, Turner JR, de Lanerolle P. Inhibiting myosin light chain kinase retards the growth of mammary and prostate cancer cells. Eur J Cancer 2006; 42: 948-57.

7 Fazal F, Gu L, Innatovych I, Han Y, Hu W, Antic N, et al. Inhibiting myosin light chain kinase induces apoptosis in vitro and in vivo. Mo Cell Biol 2005; 25: 6259-66.

8 Diecke FP, Wen Q, Iserovich P, Li J, Kuang K, Fischbarg J. Regulation of $\mathrm{Na}-\mathrm{K}-2 \mathrm{Cl}$ cotransport in cultured bovine corneal endothelial cells. Exp Eye Res 2005; 80: 777-85.

9 Korzeniewska-Dyl I. Caspases--structure and function. Pol Merkur Lekarski 2007; 23: 403-7.

10 Mehlen P, Puisieux A. Metastasis: a question of life or death. Nat Rev Cancer 2006; 6: 449-58.

11 Schultz DR, Harrington WJ Jr. Apoptosis: programmed cell death at a molecular level. Semin Arthritis Rheum 2003; 32: 345-69.

12 Maruyama J, Naguro I, Takeda K, Ichijo H. Stress-activated MAP kinase cascades in cellular senescence. Curr Med Chem 2009; 16: 1229-35.

13 Rincon M, Davis RJ. Regulation of the immune response by stressactivated protein kinases. Immunol Rev 2009; 228: 212-24.

14 Werlen G, Hausmann B, Naeher D, Palmer E. Signaling life and death in the thymus: timing is everything. Science 2003; 299: 1859-63.

15 Wada T, Penninger JM. Mitogen-activated protein kinases in apoptosis regulation. Oncogene 2004; 23: 2838-49.

16 Dent P, Yacoub A, Fisher PB, Hagan MP, Grant S. MAPK pathways in radiation responses. Oncogene 2003; 22: 5885-96.

17 Hommes DW, Peppelenbosch MP, van Deventer SJ. Mitogen activated protein (MAP) kinase signal transduction pathways and novel anti- inflammatory targets. Gut 2003; 52: 144-51.

18 You J, Mi D, Zhou X, Qiao L, Zhang H, Zhang X, et al. A positive feedback between activated extracellularly regulated kinase and cyclooxygenase/lipoxygenase maintains proliferation and migration of breast cancer cells. Endocrinology 2009; 150: 1607-17.

19 Wang FZ, Sha L, Zhang WY, Wu LY, Qiao L, Li N, et al. Involvement of hepatitis B X-interacting protein (HBXIP) in proliferation regulation of cells. Acta Pharmacol Sin 2007; 28: 431-8.

20 Qiao L, Zhao TJ, Wang FZ, Shan CL, Ye LH, Zhang XD. NF-kappaB downregulation may be involved the depression of tumor cell proliferation mediated by human mesenchymal stem cells. Acta Pharmacol Sin 2008; 29: 333-40.

21 Zhou XL, Qin XR, Zhang XD, Ye LH. Downregulation of Dickkopf-1 is responsible for high proliferation of breast cancer cells via losing control of Wnt/beta-catenin signaling. Acta Pharmacol Sin 2010; 31: 202-10.

22 Zhou X, Liu Y, You J, Zhang H, Zhang X, Ye L. Myosin light-chain kinase contributes to the proliferation and migration of breast cancer cells through cross-talk with activated ERK1/2. Cancer Lett 2008; 270: 312-27.

23 Beresford MJ, Wilson GD, Makris A. Measuring proliferation in breast cancer: practicalities and applications. Breast Cancer Res 2006; 8: 216.

24 Anand S, Penrhyn-Lowe S, Venkitaraman AR. AURORA-A amplification overrides the mitotic spindle assembly checkpoint, inducing resistance to Taxol. Cancer Cell 2003; 3: 51-62.

25 Eferl R, Wagner EF. AP-1: a double-edged sword in tumorigenesis. Nat Rev Cancer 2003; 3: 859-68.

26 Hermeking H. The 14-3-3 cancer connection. Nat Rev Cancer 2003; 3 : 931-43.

27 Ye LH, Wu LY, Guo W, Ma HT, Zhang XD. Screening of a sub-clone of human breast cancer cells with high metastasis potential. Zhonghua Yi Xue Za Zhi 2006; 86: 61-5.

28 Zhao Y, Chen X, Cai L, Yang Y, Sui G, Wu J. Angiotensin II suppresses adriamycin-induced apoptosis through activation of phosphatidylinositol 3-kinase/Akt signaling in human breast cancer cells. Acta Biochim Biophys Sin 2008; 40: 304-10.

29 Tsuchiya T, Tsuno NH, Asakage M, Yamada J, Yoneyama S, Okaji Y, et al. Apoptosis induction by $\mathrm{p} 38 \mathrm{MAPK}$ inhibitor in human colon cancer cells. Hepatogastroenterology 2008; 55: 930-5.

30 Makin G, Dive C. Modulating sensitivity to drug-induced apoptosis: the future for chemotherapy? Breast Cancer Res 2001; 3: 150-3.

31 lyoda K, Sasaki Y, Horimoto M, Toyama T, Yakushijin T, Sakakibara $\mathrm{M}$, et al. Involvement of the p38 mitogen-activated protein kinase cascade in hepatocellular carcinoma. Cancer 2003; 97: 3017-26. 